\title{
A comparative analysis of protocols for detection of $T$ cell clonality in formalin-fixed, paraffin-embedded tissue-implications for practical use
}

\author{
Irina Bonzheim • Florian Fröhlich • Patrick Adam • \\ Sema Colak • Gisela Metzler • \\ Leticia Quintanilla-Martinez • Falko Fend
}

Received: 31 August 2011 /Accepted: 22 November 2011 / Published online: 10 December 2011

(C) Springer-Verlag 2011

\begin{abstract}
Analysis of T cell receptor (TCR) gene rearrangements is an important tool for the diagnosis of $\mathrm{T}$ cell nonHodgkin lymphomas (NHL). A number of PCR-based T cell clonality protocols with increasing complexity in primer design have been published in the last decades. The multiplex TCR $\gamma$ and TCR $\beta$ assays developed by the BIOMED-2 consortium have shown superior sensitivity for the detection of clonality in T-NHL. However, they have mainly been tested on fresh frozen tissues and may be difficult to interpret due to their complex design with multiple product size ranges. In this study, two relatively simple, first-generation TCR $\gamma$ PCR protocols published by McCarthy et al. (Diagn Mol Pathol 1:173-179, 1992) and Trainor et al. (Blood $78: 192-196,1991)$ were compared with the BIOMED-2 $\operatorname{TCR} \gamma$ and TCR $\beta$ assays, using fluorescence-labeled primers and GeneScan analysis. FFPE samples of 52 peripheral T-NHL and 55 controls, including 20 B-NHL, were included. A 50-case subset including all samples with false negative or false positive results with the two TCR $\gamma$ protocols was analysed additionally with BIOMED-2 TCR $\gamma$ and TCR $\beta$ assays. With the combined BIOMED-2 assays, clonality was detected in four out of six previously false negative T-NHL and
\end{abstract}

Authors Irina Bonzheim and Florian Fröhlich contributed equally.

I. Bonzheim · F. Fröhlich · P. Adam · S. Colak ·

L. Quintanilla-Martinez $\cdot$ F. Fend $(\triangle)$

Institute of Pathology, University Hospital and Comprehensive

Cancer Center Tübingen,

Liebermeisterstr. 8,

72076 Tübingen, Germany

e-mail: falko.fend@med.uni-tuebingen.de

G. Metzler

Department of Dermatology, University Hospital Tübingen,

Tübingen, Germany increased sensitivity for this selected subgroup to $92 \%$, as compared to $64 \%$ (McCarthy) and $72 \%$ (Trainor). The overall specificity of $80 \%$ for BIOMED-2 assays was comparable to Trainor (84\%) and McCarthy (88\%) protocols, but incomplete TCR $\beta$ DJ rearrangements were identified in four out of ten BNHL cases. In conclusion, BIOMED-2 TCR $\gamma$ and TCR $\beta$ assays show superior sensitivity for the detection of $\mathrm{T}$ cell clonality. However, the complexity of BIOMED-2 protocols requires stringent quality control and experience in interpreting GeneScan patterns.

Keywords T cell clonality · Formalin-fixed paraffin-embedded tissue $\cdot$ BIOMED-2

\section{Introduction}

The histopathological diagnosis of lymphoid malignancies can be challenging, particularly when morphological, clinical and immunophenotypic findings are not conclusive. Early stages of malignant lymphoproliferations, clinical and morphological overlap with reactive conditions, absence of defining immunophenotypic abnormalities, and increasingly small diagnostic biopsy samples are common problems. Especially in peripheral $\mathrm{T}$ cell lymphomas, morphological heterogeneity, lack of easily identifiable immunophenotypical markers of clonality and high numbers of accompanying reactive cells increase the diagnostic challenge. Therefore, molecular analysis of $\mathrm{T}$ cell receptor gene rearrangements is a crucial part in the diagnostic process of suspected $\mathrm{T}$ cell lymphoproliferations.

Routine clonality assessment should be simple and robust, with good sensitivity and specificity rates. PCR-based techniques provide high sensitivity, speed and cost effectiveness, 
and can be applied to small amounts of DNA derived from formalin-fixed paraffin-embedded (FFPE) samples. For these reasons, PCR-based clonality analysis has largely replaced the previous gold standard Southern blot in daily practice [3]. However, the question which of the many published protocols for $\mathrm{T}$ cell clonality determination is best suited for routine diagnostics of FFPE tissues is far from settled.

To assess $\mathrm{T}$ cell clonality, the $\mathrm{T}$ cell receptor $\gamma$ gene (TCR $\gamma$ ) locus on $7 \mathrm{p} 14$ has been the preferential target for clonality analysis due to its restricted germline repertoire, containing nine functional variable $(\mathrm{V})$ region genes and five joining genes, which limits the number of required PCR primers, as compared to the complex TCR $\alpha$ and TCR $\beta$ gene loci. Since the 1990s, multiple protocols have been developed for the detection of TCR $\gamma$ rearrangements, ranging from relatively simple protocols with consensus primers amplifying the different $\mathrm{V} \gamma$ families in two PCRs with one product size distribution of TCR $\gamma$ rearrangements, to multiple tubes with one or more fluorochromes for primer labelling and several size ranges for amplified polyclonal products [1, 2, 4-8]. Although the use of multiple primer sets covering most or all $\mathrm{V} \gamma$ genes increases sensitivity, multiple ranges of product sizes may lead to small pseudoclonal peaks in a reduced polyclonal background if uncommonly used variable or joining gene segments are rearranged [9]. Furthermore, the number of primers in a single tube reaction may also affect results and can lead to false negativity as a result of competitive inhibition of amplification in the multiplex setting [10].

Although the high complexity of the TCR $\beta$ gene proved to be challenging for primer design, several groups have undertaken to develop protocols to detect TCR $\beta$ rearrangements with a variety of approaches, mostly using highly degenerate primers [10-15]. This was shown to increase the sensitivity and reliability of $\mathrm{T}$ cell clonality detection when compared to TCR $\gamma$-based protocols $[11,16]$.

A major effort to improve and standardize protocols for clonality determination was undertaken by the BIOMED-2 concerted action BMH4-CT98-3936 which included more than 30 European laboratories and large numbers of samples. In addition to PCR assays for the TCR $\gamma$ locus, primer sets for the TCR $\beta$ and TCR $\delta$ loci have been included. Since publication of the initial results [10] and commercialization of the primer sets, a multitude of further studies regarding the utility of the BIOMED-2 assays for $\mathrm{T}$ cell clonality analysis with freshly frozen and FFPE samples have been published. Although excellent sensitivity was demonstrated in most studies, the aspect of false positive results, which may have far-reaching clinical implications, has received considerably less attention [9, 17]. Several aspects of the BIOMED-2 assays may affect performance in daily practice. BIOMED-2 assays were developed on freshly frozen tissue $[10,18]$. Although they have by now been tested extensively in FFPE tissues [17, 19], their complex design with several products distributed over a relatively large size range increases the risk for both overcalling clonality based on preferential amplification due to poor template quality, as well as false negative results because of dropout of larger product sizes. For PCRs targeting loci with limited germ line variety, separation techniques based on both product length and sequence, such as, heteroduplex analysis, are superior to methods purely separated by product size, such as, the widely used capillary electrophoresis with fluorescence-labelled primers (Genescan) [10, 20, 21]. In addition, interpretation of the complex results requires considerable knowledge of potential biological and technical pitfalls as well as significant experience of test performance under local laboratory conditions, increasing the risk for misinterpretation in less experienced laboratories [20].

The aim of this study was to compare sensitivity and specificity of two previously published, relatively simple, but broadly used TCR $\gamma$ clonality protocols of the first generation with the BIOMED-2 TCR $\gamma$ and TCR $\beta$ assays in a panel of well-characterized peripheral $\mathrm{T}$ cell lymphomas and control cases $[1,2,10]$. Using capillary electrophoresis with fluorochrome-labelled primers as detection method, we specifically wanted to test in a routine setting, whether the practical benefit of the increased sensitivity of the BIOMED-2 protocols might be affected by a higher rate of false positive or equivocal results, as has been suggested by others [9]. We therefore enriched our samples for problematic, but nevertheless, commonly investigated tissue types, such as, skin and bone marrow biopsies, and also included a panel of B cell lymphomas.

\section{Materials and methods}

\section{Samples}

A total of 52 cases of morphologically and phenotypically well-characterized, paraffin-embedded peripheral $\mathrm{T}$ cell lymphomas (PTCL) and 55 control cases, including, $25 \mathrm{~B}$ cell non-Hodgkin lymphomas (B-NHL) and 30 reactive lesions were retrieved from the Institute of Pathology and the Department of Dermatology, University Hospital Tübingen, Germany. Specific diagnoses and tissue sources are listed in Table 1. All lymphoma cases were classified according to the guidelines of the World Health Organization Classification of Tumors of Hematopoietic and Lymphoid Tissues [22] and reviewed by three of the authors (P. A., L.Q.-M., F.F.). Special emphasis was given to smaller samples with limited numbers of lymphocytes, such as, bone marrow $(n=23)$ and skin $(n=21)$ biopsies. Bone marrow biopsies were fixed in formalin, decalcified with EDTA 
Table 1 Specific diagnoses and tissue sources
$A L K$ anaplastic lymphoma

kinase, $C L L$ chronic

lymphocytic leukemia, $E B V$

Epstein-Barr virus

\begin{tabular}{|c|c|c|c|c|}
\hline Diagnosis & Sample origin & $\begin{array}{l}\text { Samples in study } \\
\text { set } n=107\end{array}$ & Percent & $\begin{array}{l}\text { Samples in study } \\
\text { set } n=50\end{array}$ \\
\hline
\end{tabular}

\section{T cell lymphoma}

Peripheral T cell lymphoma,

Lymphomatoid papulosis not otherwise specified

Angioimmunoblastic

$\mathrm{T}$ cell lymphoma

Mycosis fungoides

Anaplastic large cell

lymphoma, ALK positive

$\mathrm{T}$ cell large granular

lymphocytic leukaemia

Enteropathy-associated

$\mathrm{T}$ cell lymphoma

Hepatosplenic T cell lymphoma

Anaplastic large cell lymphoma, ALK negative

$\mathrm{T}$ cell prolymphocytic

leukaemia

Cytotoxic mucocutaneous

T cell lymphoma

Primary cutaneous cytotoxic

$\mathrm{T}$ cell lymphoma

B cell lymphoma

Follicular lymphoma

Diffuse large B cell lymphoma

CLL

EBV-positive diffuse large

B cell lymphoma

Reactive lymphoid tissues

Lymph node

Bone marrow

Skin with benign

inflammatory

dermatosis

$\begin{array}{lr}\text { Lymph node } & 12 \\ \text { Bone marrow } & 4 \\ \text { Colon } & 1 \\ \text { Bone } & 1 \\ \text { Skin } & 1 \\ \text { Lymph node } & 12 \\ \text { Skin } & 1\end{array}$

Skin

Lymph node

Bone marrow

Stomach

Small intestine

Bone marrow

Spleen

Colon

Skin

Bone marrow

Paranasal sinus

Skin

Lymph node

Bone marrow

Lymph node

Bone marrow

Bone marrow

Lymph node

Total

$19.8 \quad 7$
2
1

2

1

13

12.2

2

1.9

1 for $7 \mathrm{~h}$ and embedded in paraffin, which results in good DNA quality comparable to other FFPE tissues [23, 24]. Ethics approval for the study was obtained from the local ethics committee, University Hospital Tübingen.

DNA isolation

DNA was extracted from $10-\mu \mathrm{m}$ paraffin-embedded tissue sections. After dewaxing, standard proteinase $\mathrm{K}$ digestion was performed. DNA quality was assessed in all samples with the BIOMED-2 control gene PCR [10] and subsequent agarose gel electrophoresis. For both Trainor and McCarthy protocols, crude DNA extracts, as well as DNA extracts purified by phenol/chloroform/isoamyl alcohol were compared in their performance in a major subset of the samples, whereas BIOMED-2 assays were only performed with purified samples.

Clonality analysis

The PCR of TCR rearrangements of all 107 tissue samples was performed using TCR $\gamma$ primer sets previously described by McCarthy et al. and Trainor et al. [1, 2]. In modification of 
the original reports, however, $\mathrm{J} \gamma$ primers were modified with D4 fluorescent dyes (Sigma-Aldrich, St. Louis, MO, USA). The first approach by McCarthy et al. consists of two Vconsensus primers targeting eight $V \gamma$ genes and two consensus primers for the five $J \gamma$ genes within two tubes, but does not amplify $\mathrm{V} \gamma 9$ rearrangements. The PCR products range from 75 to 95 and 80 to 110 base pairs for the $\mathrm{J} \gamma$ and the Jp primer sets, respectively, thus making the assay well suited for FFPEderived DNA. The protocol by McCarthy et al. was adapted with an initial denaturation step of $98^{\circ} \mathrm{C}(30 \mathrm{~s}), 38$ cycles $\left(98^{\circ} \mathrm{C} 10 \mathrm{~s}, 57^{\circ} \mathrm{C} 60 \mathrm{~s}, 72^{\circ} \mathrm{C} 30 \mathrm{~s}\right)$ and a final step of $7 \mathrm{~min}$. The second TCR $\gamma$ method by Trainor et al. involves a multiplex assay with nine $\mathrm{V} \gamma$ primers and three $\mathrm{J} \gamma$ primers (D4 flourescently-labeled) which are divided into two tubes [2]. Amplification according to Trainor et al. was modified with an initial denaturation step of $98^{\circ} \mathrm{C}(30 \mathrm{~s}), 42$ cycles $\left(98^{\circ} \mathrm{C} 10 \mathrm{~s}\right.$, $55^{\circ} \mathrm{C} 60 \mathrm{~s}, 72^{\circ} \mathrm{C} 60 \mathrm{~s}$ ) and a final step of $10 \mathrm{~min}$. Phusion Hot Start DNA Polymerase (Finnzymes Oy, Espoo, Finland) was used for amplification in conjunction with Phusion GC Buffer containing $\mathrm{MgCl}_{2}$.

Each sample was analysed at least in duplicate by using two different DNA dilutions in case of unpurified DNA, or 30 and $100 \mathrm{ng}$ of purified DNA, respectively. DNA of the cell lines Jurkat and SUP-M2 were used as positive controls.

A subset of 50 samples, including 25 T-NHL and 25 control cases was analysed in duplicate for TCR $\gamma$ and TCR $\beta$ rearrangements using the commercially available BIOMED-2 assays [10] (InVivoScribe Technologies Inc., San Diego, CA) according to the manufacturer's instruction manual with the modification of using $25-\mu$ l reactions with $250 \mathrm{ng}$ of purified DNA. In these 50 case subsets, we included all samples which had rendered false negative, false positive or equivocal results with the Trainor and McCarthy TCR $\gamma$ protocols (see below). Since $\gamma \delta$-T cell neoplasms or T-lymphoblastic lymphomas were not included in the study, the BIOMED-2 TCR $\delta$ assay was not performed.

\section{GeneScan analysis}

For GeneScan analysis, $1 \mu \mathrm{l}$ of the PCR products was mixed with sample loading solution containing $0.45 \mu$ of DNA Size Standard 400 (Beckman Coulter, Brea, CA, USA). The products were separated by capillary electrophoresis on the GenomeLab GeXP Genetic Analysis System and analysed by the GenomeLab GeXP software 10.2 (Beckman Coulter, Brea, CA, USA).

Evaluation criteria and statistical analysis

Criteria of interpretation All cases were evaluated by two independent observers, and discrepancies were resolved by discussing the GeneScan images to reach consensus. The following definitions were used throughout the study:

- Clonal: one/two reproducible peaks greater than threefold the height of the neighboring peaks within the expected size range for a given primer set

- Equivocal: one or two clearly discernible and reproducible peaks, but less than threefold the height of the neighboring peaks for a given primer set within the appropriate size range

- Polyclonal: amplicons of different length and sufficient quantity covering the whole expected size range in a given primer set, with a near-Gaussian curve matching or similar to the polyclonal control

- Oligoclonal: three or more, usually not reproducible peaks within the appropriate size range and regarded as polyclonal for the purpose of the study

- Technically not evaluable: no amplification or weak amplification product with a mean fluorescence signal intensity significantly below average with few minor peaks not reproducible in repeat PCRs

\section{Results}

Samples

A total of 52 T-NHL specimens were analysed, including 19 PTCL, not otherwise specified, 13 angioimmunoblastic T cell lymphomas (AITL) and seven cases of mycosis fungoides, among others. Diagnoses and tissue sources are listed in Table 1. The control set included 25 B-NHL and 30 specimens of reactive lymphoid tissues, reactive bone marrow (BM) biopsies and skin biopsies with a variety of well-characterized benign inflammatory dermatoses. Of the samples analysed, 95/107 (89\%) showed amplification of the 300-bp band in the BIOMED-2 control PCR including all skin and BM biopsies.

Detection of clonality in primary samples with McCarthy and Trainor primer sets

All 107 cases were analysed using the protocols of McCarthy et al. and Trainor et al. Clonal TCR $\gamma$ rearrangements were detected in 42 out of $52(81 \%)$ and 43 out of $52(83 \%)$ TNHL samples using the McCarthy and Trainor protocols, respectively.

In total, 45 (86\%) samples were monoclonal with at least one technique, and concordant monoclonal results with both methods were detected in 40 out of 45 of the cases. In contrast, six (12\%) cases (four peripheral T cell lymphoma, not otherwise specified (PTCL-NOS), one AITL and one cytotoxic mucocutaneous $\mathrm{T}$ cell lymphoma) remained poly-/ 
oligoclonal with both techniques, and one case was poly-/oligoclonal with one and equivocal with the second primer set. Of the six discordant cases, three were monoclonal with the Trainor primer set and poly/-oligoclonal or equivocal with the McCarthy set. Two of these cases showed a clear-cut monoclonal result with Trainor tube 1 and a polyclonal pattern with McCarthy tube 1, indicating the presence of a $\mathrm{V} \gamma 9$ rearrangement in these cases. This was confirmed with the BIOMED-2 TCR $\gamma$ primer set (Fig. 1a). The other three discordant cases included two cases monoclonal with the McCarthy assay and polyclonal or equivocal with the Trainor primer set, and finally a case with equivocal results in McCarthy, but clearly polyclonal in the Trainor assay.

Clonal or equivocal results were identified in six out of $55(11 \%)$ control cases with the McCarthy protocol and six out of $53(11 \%)$ amplifiable controls with the Trainor assay. A single reactive $\mathrm{BM}(3 \%)$ rendered a monoclonal result with both primer sets, four (13\%) reactive cases showed a monoclonal or equivocal result with one of the two protocols, whereas the remaining benign controls remained polyclonal in all PCRs. Three (12\%) of 25 B-NHL cases showed a reproducible monoclonal TCR rearrangement with one (one case) or both (two cases, Fig. 1b) primer sets.

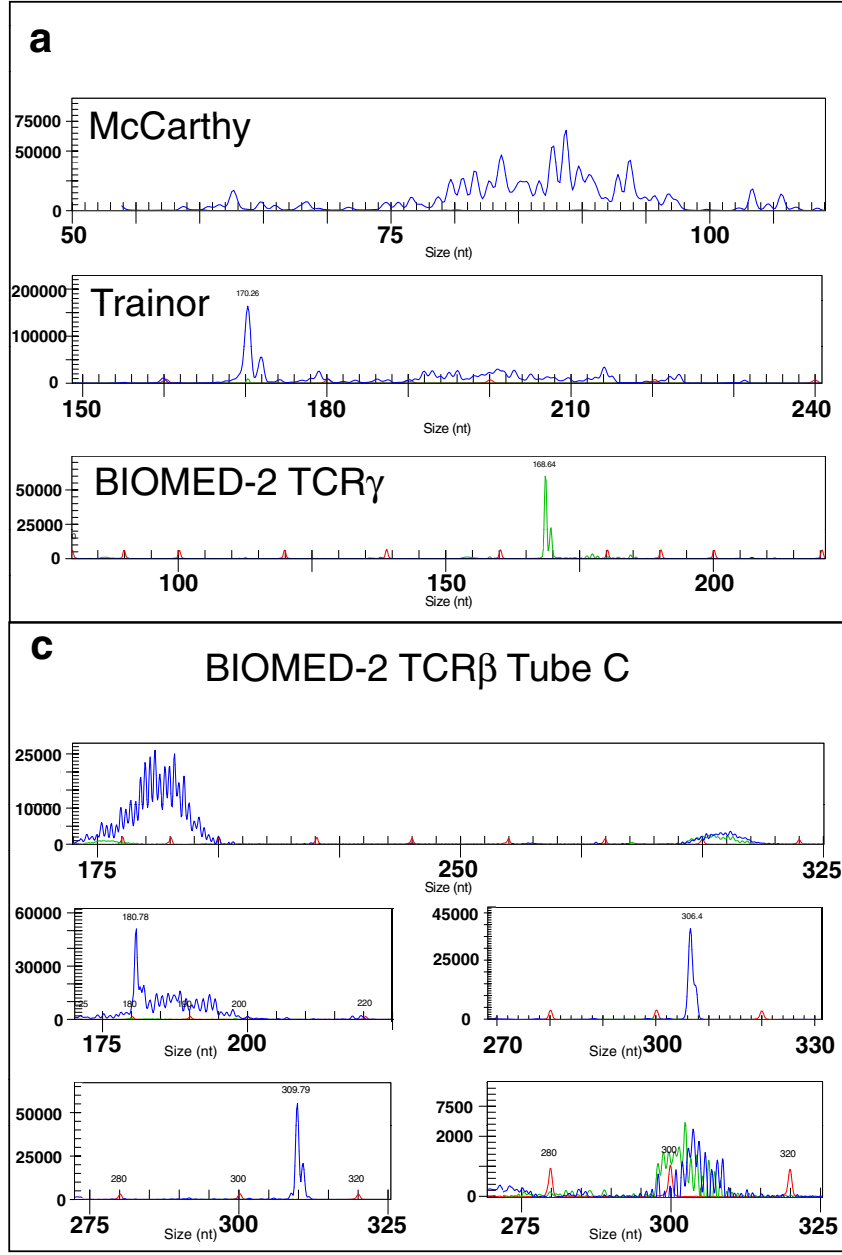

Fig. 1 GeneScan analysis of representative samples. a AITL (lymph node) with a clonal TCR $\gamma$ V9 rearrangement. There is a clear-cut clonal pattern in the Trainor primer set 1, but a polyclonal pattern in McCarthy primer set 1 . Monoclonality is confirmed with BIOMED-2 TCR $\gamma$ Tube B. b DLBCL, bone marrow biopsy. Both McCarthy and Trainor primer sets 1 show a clonal TCR $\gamma$ product with prominent polyclonal background confirmed in repeats. c Incomplete DJ $\beta$ rearrangements. Reactive lymph node, TCR $\beta$ Tube C. Although both products show a polyclonal pattern, the larger-sized product shows a significantly diminished intensity, indicating differences in amplification efficiency (upper lane). B- b
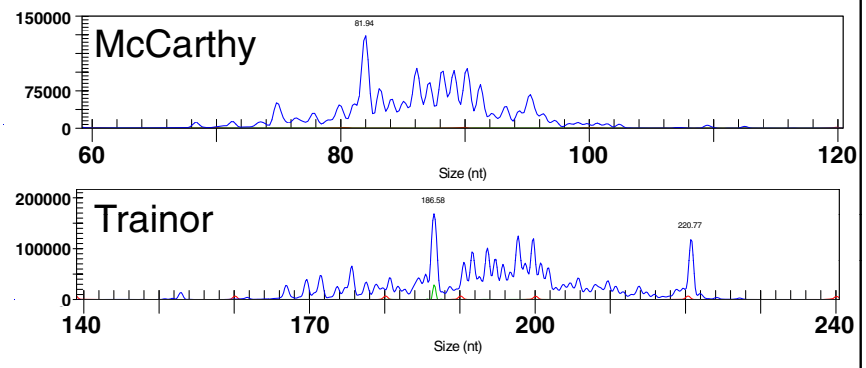

d
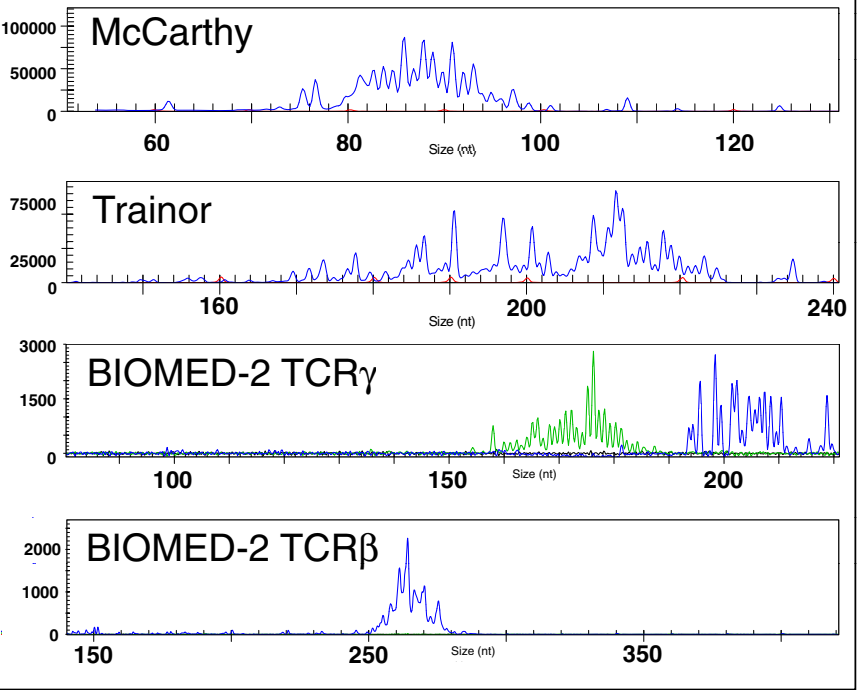

NHL with a monoclonal peak and polyclonal background in both product size ranges, indicative of an incomplete DJ $\beta$ rearrangement. TCR $\beta$ Tube $\mathrm{C}$ (middle lane). Two PTCL with monoclonal (left) and polyclonal (right) PCR products (lower lane). d Skin biopsy with benign inflammatory dermatosis demonstrating polyclonal results with McCarthy and Trainor primer sets 1 , as well as the BIOMED-2 TCR $\gamma$ (Tube B) and TCR $\beta$ assays (Tube B). Note the irregular, not perfectly Gaussian distribution of products in all PCRs due to a limited number of T-cells and possibly restricted repertoire. The occasional peaks of increased intensity were not reproducible in repeats 
Comparison with BIOMED-2 TCR $\gamma$ and TCR $\beta$ protocols

From the total study set of 107 cases, a subset of 50 cases, which exhibited the required DNA quality (at least 300-bp products in the BIOMED-2 control PCR) were additionally analysed by the TCR $\gamma$ and TCR $\beta$ BIOMED- 2 assays (Table 1). This subset contained 25 peripheral $\mathrm{T}$ cell lymphomas, including all six cases, which had shown a polyclonal pattern using the Trainor and McCarthy protocols, and the two T-NHLs with suspected $\mathrm{V} \gamma 9$ rearrangements. Additionally, 25 controls including most samples giving monoclonal or equivocal results were analysed. In this subset of 50 "difficult" cases specifically selected to test the performance of the BIOMED-2 protocols, the McCarthy primer set achieved a sensitivity of $64 \%$ (16 out of 25 ), whereas a sensitivity of $72 \%(18 / 25)$ was reached with the Trainor protocol. Detailed results are shown in Fig. 2a, b.

Of the 25 T-NHL cases, 23 showed a clonal result with one or both BIOMED-2 sets (TCR $\gamma$ or TCR $\beta$ ), including all cases found to be monoclonal with one or both previous techniques. Four of the six polyclonal cases with both Trainor and McCarthy primer sets revealed a monoclonal product with the TCR $\beta$ set, including one with incomplete $\mathrm{DJ} \beta$ rearrangement only (Tube $C$ ), increasing the sensitivity for the combined BIOMED-2 assays to $92 \%$. One of these cases additionally revealed a TCR $\gamma$ rearrangement.

Of the 15 reactive control cases analysed, four $(27 \%)$ cases showed either a monoclonal (one sample), equivocal (one sample) or technically not evaluable result (two samples) with the combined BIOMED-2 TCR $\gamma$ and TCR $\beta$ assays. In comparison, three out of $15(20 \%)$ cases had shown a monoclonal or equivocal result with the Trainor or McCarthy protocols, of which one proved to be polyclonal with both BIOMED-2 sets. Of the ten B-NHL samples analysed with BIOMED-2 primers, one showed an equivocal result with the TCR $\gamma$ set, and four monoclonal results with the TCR $\beta$ set. Of interest, all four samples exhibited only an incomplete $\mathrm{DJ} \beta$ rearrangement (Tube C) (Fig. 1c). In contrast, three out of ten B-NHL samples showed monoclonal or equivocal results with both McCarthy and Trainor protocols. The results of the 50-case subset with BIOMED-2 primers are depicted in Fig. 2c-e. Specificities in the BNHL cases were $80 \%$ (McCarthy), 70\% (Trainor) and 60\% (BIOMED-2 TCR $\gamma$ and TCR $\beta$ ) versus $93 \%$ (all protocols) for reactive cases, if equivocal cases were considered together with the poly-/oligoclonal cases. For all non-T-NHL cases combined, specificity was $88 \%$ (McCarthy) versus $84 \%$ (Trainor) and $80 \%$ (BIOMED-2 TCR $\gamma$ and TCR $\beta$ ).

In summary, the combined BIOMED-2 TCR $\gamma$ and TCR $\beta$ assays revealed a superior sensitivity of $92 \%$ and a comparable specificity of $80 \%$ as compared to the McCarthy and Trainor protocols.
Influence of tissue type, DNA quality and DNA purification

The specimen type was correlated with the PCR results to identify a potential influence of the tissue source. In the 50case subset, false negative results were overrepresented among lymph node biopsies with McCarthy, Trainor and BIOMED-2 TCR $\gamma$ protocols.

The false positive results among control cases in general were overrepresented among bone marrow biopsies. Equivocal results were observed with all protocols among reactive BM biopsies, whereas no equivocal results were found among the lymph node samples, indicating an influence of DNA quality and number of lymphocytes in the sample.

Suboptimal DNA quality, defined as showing only the 200-bp band in the control PCR, was present in 12 out of 107 samples, including six T-NHL and six control samples. Both McCarthy and Trainor protocols detected a monoclonal rearrangement in five out of six T-NHLs. Furthermore, a polyclonal result was obtained in all six control samples with the McCarthy protocol and in five out of six with the Trainor primer sets. These results are similar to the whole collective, indicating relatively robust assay performance. These samples, however, were considered inadequate according to guidelines for the BIOMED-2 primer sets and therefore not analysed further.

Purified and unpurified DNA extracts were compared with Trainor and McCarthy protocols. An improvement in assay performance was noted in a third of the investigated samples after purification.

\section{Discussion}

Detection of $\mathrm{T}$ cell clonality in clinical samples is an important tool for the diagnosis of $\mathrm{T}$ cell lymphoproliferative disorders. Consequently, a large number of PCR tests for this purpose have been developed, culminating in the multinational and multi-institutional effort, which resulted in the development of the BIOMED-2 protocols. These assays for the detection of T cell clonality targeting both TCR $\gamma$ and TCR $\beta$ loci show superior sensitivity compared with most previously published protocols [10]. Clonality detection rates in T-NHL of $86-89 \%, 87-94 \%$ and $94-100 \%$ were reported for the TCR $\gamma, \operatorname{TCR} \beta$ and the combined assays, respectively, in freshly frozen tissues. With FFPE material, analysis of either TCR $\gamma$ or TCR $\beta$ renders monoclonal results in $69-81 \%$ of cases, reaching $80-100 \%$, when both assays are evaluated jointly [3, 10, 17, 25, 26]. Our comparative analysis confirms that the addition of BIOMED-2 primers increases the sensitivity of clonality analysis in FFPE tissues of T-NHL over simpler protocols. The 
Fig. 2 Results of TCR $\gamma$ PCR for the 50-case subset, using the modified protocols according to a McCarthy and $\mathbf{b}$ Trainor, as well as $\mathbf{c}$ BIOMED-2 TCR $\gamma$ and d BIOMED- 2 TCR $\beta$ and $\mathbf{e}$ the combined BIOMED-2 assays. Percentages of clonal, polyclonal, equivocal and technically not evaluable results are given for T-NHL, B-NHL and reactive samples. Only incomplete DJ $\mathrm{TCR} \beta$ rearrangements (Tube $\mathrm{C}$ ) were identified in B-NHL samples (asterisk) a

McCarthy 50-case subset

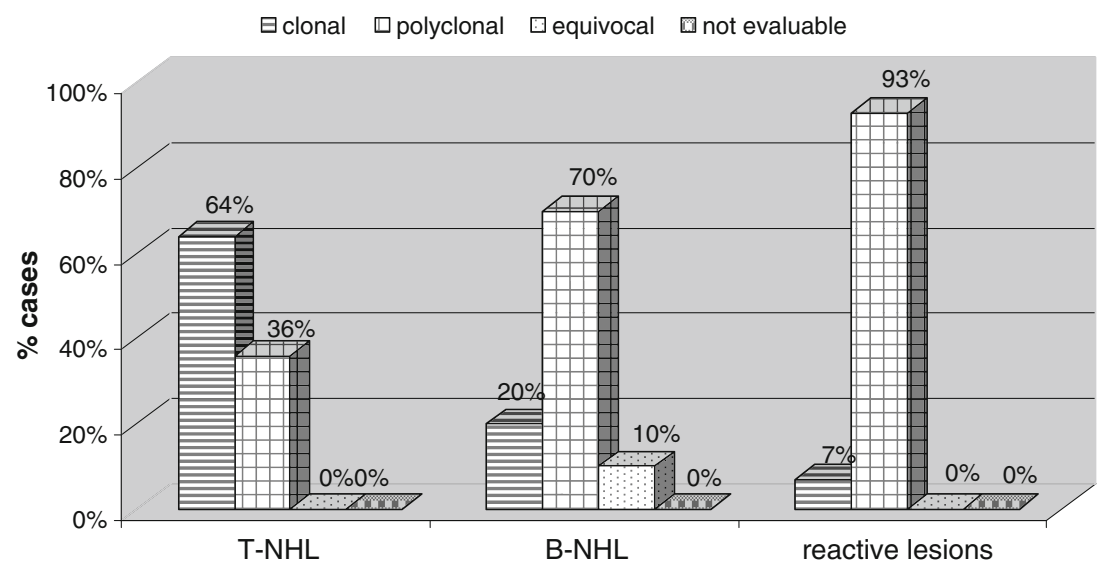

b

Trainor 50-case subset

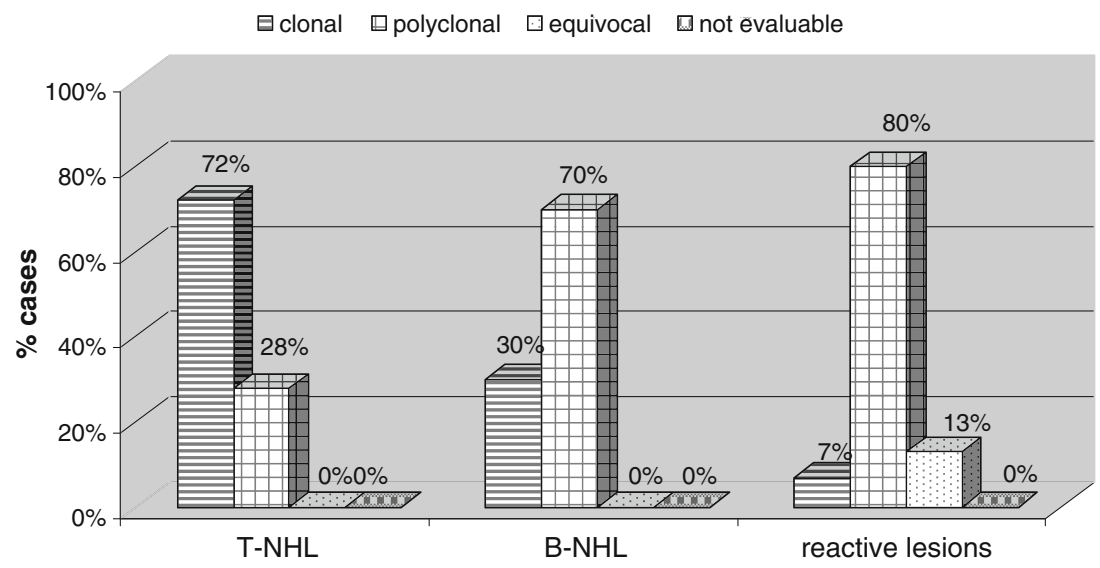

C BIOMED-2 TCRG 50-case subset

$\boxminus$ clonal $\square$ polyclonal $\square$ equivocal $\square$ not evaluable

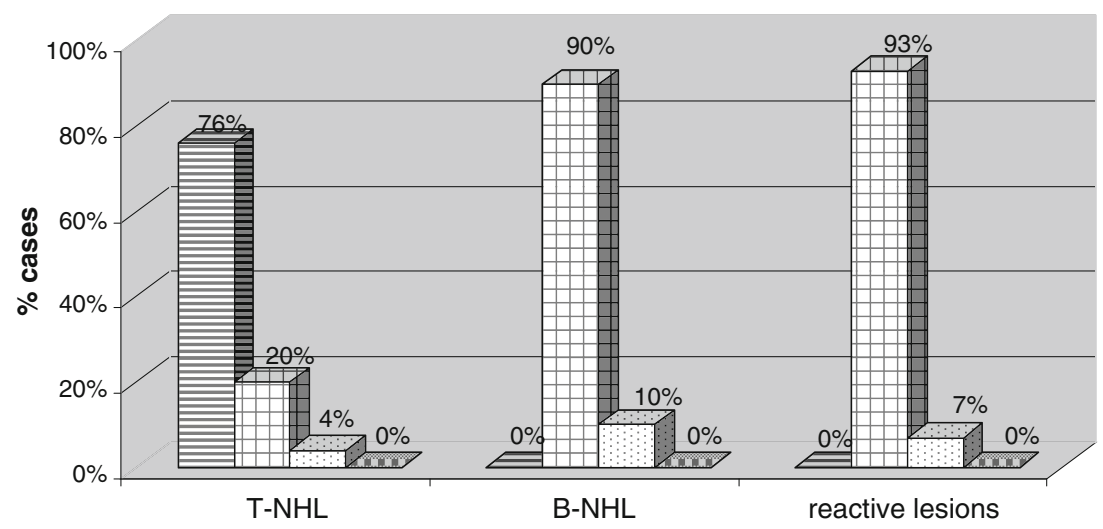

sensitivity of $92 \%$ for the combined BIOMED-2 assays in our study is remarkable because the 50 -case subset was enriched for cases negative with both Trainor and McCarthy $\mathrm{TCR} \gamma$ protocols. BIOMED-2 assays identified clonality in four of the initially false negative cases, mostly due to positive results with the TCR $\beta$ assay. Of note, the two cases which remained negative with BIOMED-2 primers corresponded to lymph node biopsies of PTCL-NOS, which showed some, but not all, diagnostic features of AITL. In contrast, all ten extranodal biopsies with T-NHL infiltrates, including skin, bone marrow and gastrointestinal tract samples rendered positive results. This indicates that the false 
Fig. 2 (continued)

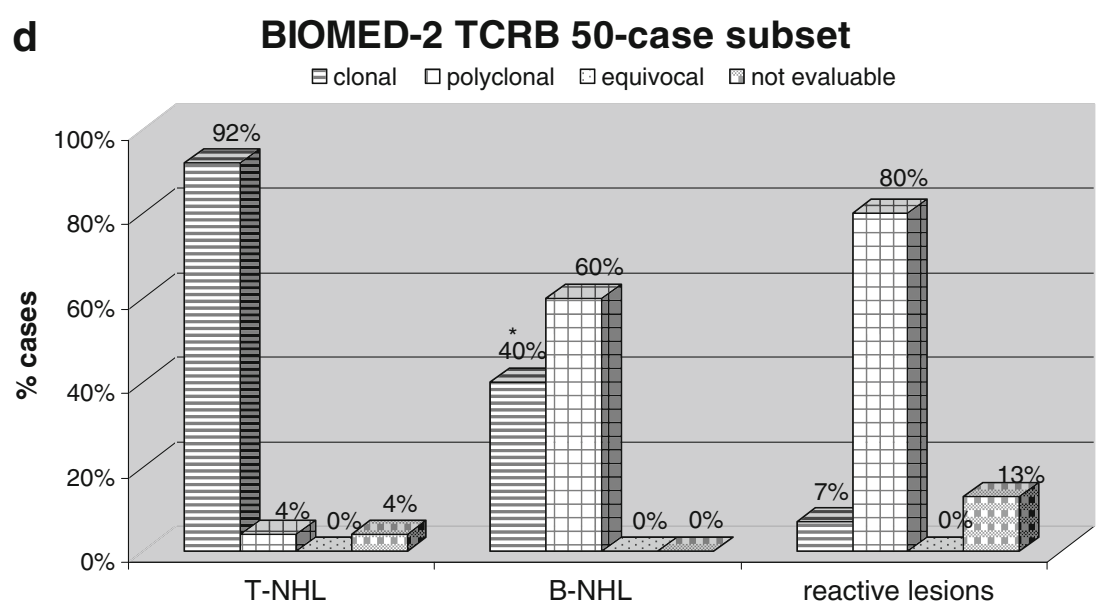

\section{e BIOMED-2 TCR combined evaluation 50-case subset}

$\boxminus$ clonal $\square$ polyclonal $\square$ equivocal $\square$ not evaluable

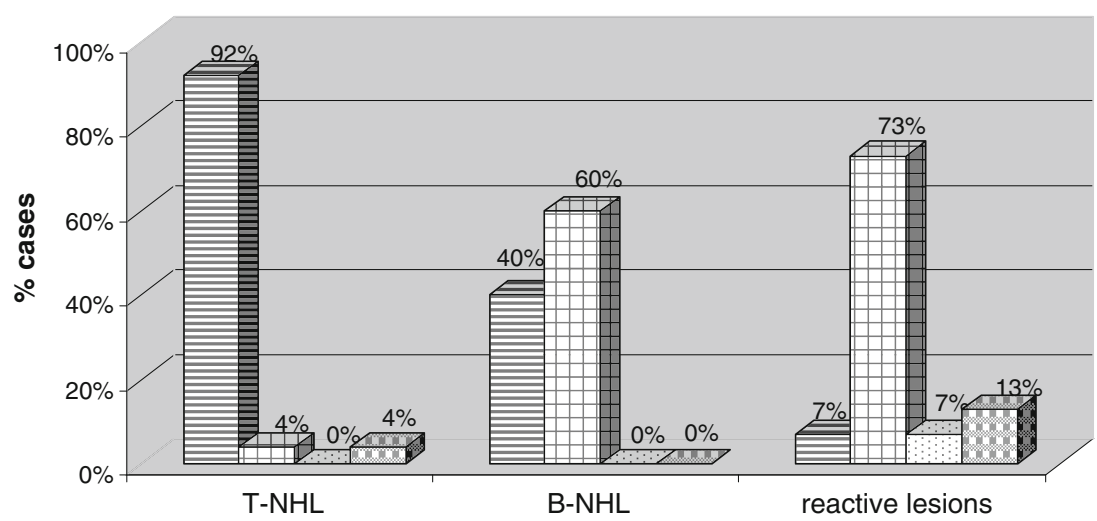

negativity was not due to poor DNA quality or biopsy size but rather to the inherent properties of the malignancy, such as, oligoclonality, which can be observed in a proportion of AITL, or presence of incomplete or deleterious rearrangements of the TCR or trans-rearrangements [27].

Another aim of our study was to investigate the issue of specificity, which has received considerably less attention in previous reports. A large study of reactive lymphoproliferations performed by the BIOMED-2 consortium analysed exclusively freshly frozen tissues and excluded FFPE tissues, stating that "PCR results of such samples are largely dependent on variation in formalin fixation and paraffin embedding" [18]. In daily practice, however, many laboratories commonly have to deal with FFPE samples. We therefore focussed on FFPE tissues and included a number of "difficult" samples, such as, EDTA-decalcified BM, endoscopic and skin biopsies, which are often overrepresented among samples submitted to clonality analysis. If only clearly reproducible false positive results are taken into account, BIOMED-2, McCarthy and Trainor protocols show roughly equal specificities ranging from $80 \%$ to $88 \%$. False positive results can be due to underlying biological as well as technical factors. On one hand, minor $\mathrm{T}$ cell clones are detectable in reactive conditions such as autoimmune diseases or viral infections [18, 28, 29]. In addition, the presence of a monoclonal $\mathrm{T}$ cell population could be due to a clinically and morphologically undetectable (pre-) neoplastic disorder, such as, an early stage of $\mathrm{T}$ cell large granular lymphocytic leukemia in bone marrow biopsies. Of interest, most false positive results obtained with BIOMED-2 assays were due to the high incidence $(40 \%)$ of reproducible, isolated incomplete $\mathrm{DJ} \beta$ rearrangements (Tube $\mathrm{C}$ ) in our mature BNHL panel. In B-NHL, the occasional presence of monoclonal $\mathrm{T}$ cell rearrangements has been described by several groups. This can either be due to cross-lineage rearrangements, which are usually found in precursor B cell neoplasms but can occasionally occur in mature B-NHL [30, 31] or may represent expansion of benign reactive $\mathrm{T}$ cell clones in response to the B-NHL. Of interest, a relatively high rate of incomplete TCR $\beta$ DJ rearrangements in B-NHL has been observed in another study [13]. Since isolated incomplete TCR $\beta$ DJ rearrangements without clonal products with other primer sets seem to be rare in T-NHL, we recommend to interpret these isolated findings with great caution. 
Although our results show a favourable rate of false positive or equivocal results in FFPE under carefully controlled conditions, if the incomplete TCR $\beta$ DJ rearrangements are excluded, in our experience, BIOMED-2 assays are nevertheless more prone to false positive results and overinterpretation for several reasons. The PCR products generated with the BIOMED-2 primers range from 240 to $325 \mathrm{bp}$ for the TCR $\beta$ set and from 80 to $255 \mathrm{bp}$ for the TCR $\gamma$ set, which is larger than most products generated with the McCarthy (75-110 bp) and Trainor (170-230 bp) protocols. In addition, BIOMED-2 assays generate different normal product size distributions within one PCR [8]. The separation of TCR $\gamma$ rearrangement amplicons into multiple size distributions with several fluorochromes and sometimes less-than-ideal Gaussian curves makes the interpretation sometimes difficult and increases the likelihood of pseudoclonal peaks and consequently false positive results [9]. This is especially true for amplification of uncommonly used joining or variable gene segments, and if the sample contains limited numbers of $\mathrm{T}$ cells or poor DNA quality. That the amount of polyclonal target cells in the specimen is a critical factor for the generation of pseudoclonal peaks is demonstrated by the higher incidence of false positive and equivocal results in bone marrow biopsies. Although identification of pseudoclonal in contrast to true monoclonal products is facilitated by the performance of repeat PCRs, some of these minor non-malignant clones can be detected reproducibly. Whether the use of alternative, sequencesensitive techniques for product separation, such as, heteroduplex analysis, allows to reduce the rate of false positive results was not examined in this study. For skin biopsies, analysis of several specimens from different sites has been recommended and can strengthen a diagnosis of cutaneous T-NHL if the same clone is detected in more than one sample [32]. However, oligoclonality or occurrence of heterogeneously distributed, different $\mathrm{T}$ cell clones has been repeatedly described in mycosis fungoides [33, 34].

In conclusion, our study confirms the superior sensitivity of the BIOMED-2 TCR $\gamma$ and TCR $\beta$ assays in the detection of T cell clonality, even in "difficult" FFPE samples. However, the uncritical use of these protocols on FFPE samples may result in an increase of false positive results. This is especially true for extranodal samples with limited amount of lymphoid cells, poor template DNA quality and lack of experience with the interpretation of the complex product patterns. Assessment of DNA quality and routine performance of duplicate reactions are mandatory to obtain reliable results. In addition, reliance on numerical thresholds such as $>3 \times$ or $>5 \times$ peak height or similar mathematical and statistical algorithms without critical observance of overall amplification efficiency on one hand, and clinicopathological features on the other hand can result in incorrect interpretations. Amount and lymphocyte content of examined tissue, DNA quantity and quality, availability of previous samples for comparative analysis, and outcome of control and duplicate reactions all need to be taken into consideration before signing out molecular results. In the case of discrepancies between molecular and histological findings, these need to be addressed in the final report [20].

In addition, our study demonstrates that fairly simple, first-generation TCR $\gamma$ assays such as the two protocols tested above show robust performance despite their lower sensitivity and still provide an acceptable alternative, e.g. for samples with poor DNA quality or as first test in a stepwise approach to $\mathrm{T}$ cell clonality analysis. Irrespective of the protocols used, determination of $\mathrm{T}$ cell clonality in FFPE samples requires stringent quality controls and careful interpretation of results with full knowledge of potential technical and biological pitfalls.

Acknowledgements This study was supported by a grant from the interdisciplinary center for clinical research (IZKF), University Hospital Tübingen.

Conflict of interest The authors declare that they have no conflict of interest.

\section{References}

1. McCarthy KP, Sloane JP, Kabarowski JH, Matutes E, Wiedemann LM (1992) A simplified method of detection of clonal rearrangements of the T-cell receptor-gamma chain gene. Diagn Mol Pathol $1: 173-179$

2. Trainor KJ, Brisco MJ, Wan JH, Neoh S, Grist S, Morley AA (1991) Gene rearrangement in B- and T-lymphoproliferative disease detected by the polymerase chain reaction. Blood 78:192-196

3. Sandberg Y, van Gastel-Mol EJ, Verhaaf B, Lam KH, van Dongen JJ, Langerak AW (2005) BIOMED-2 multiplex immunoglobulin/ T-cell receptor polymerase chain reaction protocols can reliably replace Southern blot analysis in routine clonality diagnostics. J Mol Diagn 7:495-503

4. Arber DA, Braziel RM, Bagg A, Bijwaard KE (2001) Evaluation of $\mathrm{T}$ cell receptor testing in lymphoid neoplasms: results of a multicenter study of 29 extracted DNA and paraffin-embedded samples. J Mol Diagn 3:133-140

5. Klemke CD, Dippel E, Dembinski A, Ponitz N, Assaf C, Hummel $\mathrm{M}$ et al (2002) Clonal T cell receptor gamma-chain gene rearrangement by PCR-based GeneScan analysis in the skin and blood of patients with parapsoriasis and early-stage mycosis fungoides. $\mathrm{J}$ Pathol 197:348-354

6. Lawnicki LC, Rubocki RJ, Chan WC, Lytle DM, Greiner TC (2003) The distribution of gene segments in T-cell receptor gamma gene rearrangements demonstrates the need for multiple primer sets. J Mol Diagn 5:82-87

7. Shadrach B, Warshawsky I (2004) A comparison of multiplex and monoplex T-cell receptor gamma PCR. Diagn Mol Pathol 13:127134

8. Vega F, Medeiros LJ, Jones D, Abruzzo LV, Lai R, Manning J et al (2001) A novel four-color PCR assay to assess T-cell receptor gamma gene rearrangements in lymphoproliferative lesions. Am J Clin Pathol 116:17-24 
9. Cushman-Vokoun AM, Connealy S, Greiner TC (2010) Assay design affects the interpretation of T-cell receptor gamma gene rearrangements: comparison of the performance of a one-tube assay with the BIOMED-2-based TCRG gene clonality assay. J Mol Diagn 12:787-796

10. van Dongen JJ, Langerak AW, Bruggemann M, Evans PA, Hummel M, Lavender FL et al (2003) Design and standardization of PCR primers and protocols for detection of clonal immunoglobulin and T-cell receptor gene recombinations in suspect lymphoproliferations: report of the BIOMED-2 Concerted Action BMH4CT98-3936. Leukemia 17:2257-2317

11. Assaf C, Hummel M, Dippel E, Goerdt S, Muller HH, Anagnostopoulos I et al (2000) High detection rate of T-cell receptor beta chain rearrangements in T-cell lymphoproliferations by family specific polymerase chain reaction in combination with the GeneScan technique and DNA sequencing. Blood 96:640-646

12. Assaf C, Hummel M, Steinhoff M, Geilen CC, Orawa H, Stein H et al (2005) Early TCR-beta and TCR-gamma PCR detection of Tcell clonality indicates minimal tumor disease in lymph nodes of cutaneous T-cell lymphoma: diagnostic and prognostic implications. Blood 105:503-510

13. Dictor M, Warenholt J, Isinger A (2005) Resolving T-cell receptor clonality in two and genotype in four multiplex polymerase chain reactions. Haematologica 90:1524-1532

14. Kneba M, Bolz I, Linke B, Hiddemann W (1995) Analysis of rearranged T-cell receptor beta-chain genes by polymerase chain reaction (PCR) DNA sequencing and automated high resolution PCR fragment analysis. Blood 86:3930-3937

15. McCarthy KP, Sloane JP, Kabarowski JH, Matutes E, Wiedemann LM (1991) The rapid detection of clonal T-cell proliferations in patients with lymphoid disorders. Am J Pathol 138:821-828

16. Droese J, Langerak AW, Groenen PJ, Bruggemann M, Neumann P, Wolvers-Tettero IL et al (2004) Validation of BIOMED-2 multiplex PCR tubes for detection of TCRB gene rearrangements in Tcell malignancies. Leukemia 18:1531-1538

17. Liu H, Bench AJ, Bacon CM, Payne K, Huang Y, Scott MA et al (2007) A practical strategy for the routine use of BIOMED-2 PCR assays for detection of B- and T-cell clonality in diagnostic haematopathology. Br J Haematol 138:31-43

18. Langerak AW, Molina TJ, Lavender FL, Pearson D, Flohr T, Sambade C et al (2007) Polymerase chain reaction-based clonality testing in tissue samples with reactive lymphoproliferations: usefulness and pitfalls. A report of the BIOMED-2 Concerted Action BMH4-CT98-3936. Leukemia 21:222-229

19. Lukowsky A, Muche JM, Mobs M, Assaf C, Humme D, Hummel $\mathrm{M}$ et al (2010) Evaluation of T-cell clonality in archival skin biopsy samples of cutaneous T-cell lymphomas using the biomed-2 PCR protocol. Diagn Mol Pathol 19:70-77

20. Groenen PJ, Langerak AW, van Dongen JJ, van Krieken JH (2008) Pitfalls in TCR gene clonality testing: teaching cases. J Hematop 1:97-109

21. van Krieken JH, Langerak AW, Macintyre EA, Kneba M, Hodges E, Sanz RG et al (2007) Improved reliability of lymphoma diagnostics via PCR-based clonality testing: report of the BIOMED-2 Concerted Action BHM4-CT98-3936. Leukemia 21:201-206

22. Swerdlow S, Campo E, Harris N, Jaffe E, Pileri S, Stein H et al (2008) World Health Organization Classification of Tumours of haematopoietic and lymphoid tissues. IARC Press, Lyon

23. Fend F, Bock O, Kremer M, Specht K, Quintanilla-Martinez L (2005) Ancillary techniques in bone marrow pathology: molecular diagnostics on bone marrow trephine biopsies. Virchows Arch 447:909-919

24. Fend F, Tzankov A, Bink K, Seidl S, Quintanilla-Martinez L, Kremer $M$ et al (2008) Modern techniques for the diagnostic evaluation of the trephine bone marrow biopsy: methodological aspects and applications. Prog Histochem Cytochem 42:203-252

25. Bruggemann M, White H, Gaulard P, Garcia-Sanz R, Gameiro P, Oeschger S et al (2007) Powerful strategy for polymerase chain reaction-based clonality assessment in T-cell malignancies Report of the BIOMED-2 Concerted Action BHM4 CT98-3936. Leukemia 21:215-221

26. Patel KP, Pan Q, Wang Y, Maitta RW, Du J, Xue X et al (2010) Comparison of BIOMED-2 versus laboratory-developed polymerase chain reaction assays for detecting T-cell receptor-gamma gene rearrangements. J Mol Diagn 12:226-237

27. Yoshikai Y, Toyonaga B, Koga Y, Kimura N, Griesser H, Mak TW (1987) Repertoire of the human T cell gamma genes: high frequency of nonfunctional transcripts in thymus and mature T cells. Eur J Immunol 17:119-126

28. Hodges E, Krishna MT, Pickard C, Smith JL (2003) Diagnostic role of tests for T cell receptor (TCR) genes. J Clin Pathol 56:1-11

29. Terhune MH, Cooper KD (1993) Gene rearrangements and T-cell lymphomas. Arch Dermatol 129:1484-1490

30. Szczepanski T, Langerak AW, van Dongen JJ, van Krieken JH (1998) Lymphoma with multi-gene rearrangement on the level of immunoglobulin heavy chain, light chain, and T-cell receptor beta chain. Am J Hematol 59:99-100

31. van der Velden VH, Bruggemann M, Hoogeveen PG, de Bie M, Hart PG, Raff T et al (2004) TCRB gene rearrangements in childhood and adult precursor-B-ALL: frequency, applicability as MRD-PCR target, and stability between diagnosis and relapse. Leukemia 18:1971-1980

32. Thurber SE, Zhang B, Kim YH, Schrijver I, Zehnder J, Kohler S (2007) T-cell clonality analysis in biopsy specimens from two different skin sites shows high specificity in the diagnosis of patients with suggested mycosis fungoides. J Am Acad Dermatol 57:782-790

33. Vega F, Luthra R, Medeiros LJ, Dunmire V, Lee SJ, Duvic M et al (2002) Clonal heterogeneity in mycosis fungoides and its relationship to clinical course. Blood 100:3369-3373

34. Ponti R, Fierro MT, Quaglino P, Lisa B, Paola FC, Michela O et al (2008) TCRgamma-chain gene rearrangement by PCR-based GeneScan: diagnostic accuracy improvement and clonal heterogeneity analysis in multiple cutaneous T-cell lymphoma samples. J Invest Dermatol 128:1030-1038 